\title{
EXERCISE PROTOCOL FOR SHOULDER MOVEMENT WITH RESTRICTED AMPLITUDE MOVEMENT FOR 15 OR 30 DAYS AFTER BREAST-CONSERVING SURGERY WITH ONCOPLASTIC TECHNIQUE: RANDOMIZED CLINICAL TRIAL
}

Samantha Karlla Lopes de Almeida Rizzi', Cinira Assad Simão Haddad', Simone Elias', Afonso Celso Pinto Nazário1', Gil Facina ${ }^{1}$

'Universidade Federal de São Paulo - São Paulo (SP), Brazil.

Introduction: Early exercises in the postoperative period of breast cancer are important to prevent motor and functional complications 1,2. However, there are no prospective studies with protocols including specific exercises for patients submitted to breast oncoplastic surgery. Objectives: To assess the amplitude of movement, pain and upper limb function; incidence of dehiscence, seroma, infection and necrosis; and occurrence of reoperation in women in the postoperative period of oncoplastic breast cancer surgery, who underwent protocol of postoperative exercises with restricted shoulder movement for 15 or 30 days. Methodology: We included 60 women with breast cancer submitted to conserving surgery using the oncoplastic technique. They were assessed in the preoperative period and 07, 15, 30, 60 and 90 days after surgery. On the day after the surgery, all patients initiated an exercise protocol limited to 90 degrees, guided by the physical therapist, and performed at home. Two weeks after the surgery, they were randomized in two groups: Amplitude Free Group (30 patients) - liberation of shoulder joint amplitude at the pain threshold or until the sensation of displacement in surgical borders; Limited Amplitude Group (30 patients) - maintenance of shoulder movement restricted to $90^{\circ}$ until 30 days after the surgery, when they were also cleared to do free amplitude exercises. Results: there were no differences between groups regarding shoulder joint amplitude, pain and function of upper limbs. In the intragroup analysis, only the Limited Amplitude Group presented upper limb functional index worse than in the preoperative period. There were no differences between groups regarding the incidence of postoperative scar complications nor the need for reoperation. Conclusion: the free amplitude exercises 15 days after the surgery did not have an impact on the movement amplitude nor on the pain, with beneficial effect only in the intragroup analysis, regarding upper limb function. It was considered safe in relation to scar complications. 